In this issue

Encapsulated Fat Necrosis after Lipolysis of the Calf with Phosphatidylcholine .......................... 180

Erythema-Multiforme-Like Eruption Recurring in Ultraviolet-Exposed Skin

Symmetrical Drug-Related Intertriginous and Flexural Exanthema ................................... 183

Symmetrical Drug-Related Intertriginous and Flexural Exanthema-Reply

Dermatology 2008;216:180-181

DOI: 10.1159/000111519

\section{Encapsulated Fat Necrosis after Lipolysis of the Calf with Phosphatidylcholine}

F.G. Bechara ${ }^{\text {a }}$, D. Georgas ${ }^{\text {a }}$, M. Sand ${ }^{\text {b }}$, N. Tomi ${ }^{\text {a }}$, P. Altmeyer ${ }^{\text {a }}$, K. Hoffmann ${ }^{\text {a }}$

${ }^{a}$ Department of Dermatology and Allergology, Ruhr University Bochum, and bepartment of General and Visceral Surgery, Augusta-Krankenanstalt, Academic Teaching Hospital, Ruhr University Bochum, Bochum, Germany

\section{Key Words}

Fat necrosis, encapsulated $\cdot$ Lipolysis $\cdot$ Phosphatidylcholine

Attempts of nonsurgical reduction of localized fat accumulations by subcutaneous injections of phosphatidylcholine (PPC)containing substances have become a popular procedure in cosmetic medicine, although the definite mechanism of action, possible complications and the adequate amount of injection volume remain unsolved [1-3]. Only temporary and mild side effects induced by the so-called injection lipolysis have been reported to date [1].

In the following we report for the first time a 27 -year-old female patient who developed a persistent encapsulated fat necrosis on the right calf after injection lipolysis with PPC for treatment of localized fat accumulations. On clinical examination, mild to moderate fat pads were visible in the dorsal and medial area of the calves. Her medical history revealed no further complications; no permanent medication was reported. Recent injuries, surgeries or trauma of the lower limbs were denied. Proposed liposuction of both calves as first-line therapy was refused by the patient. We therefore decided to initiate a treatment attempt of localized fat dissolving with intralesional application of PPC. The patient received subcutaneous injections of $5 \mathrm{ml}$ PPC (Lipostabil ${ }^{\circledR}, \mathrm{MCM}$ Klosterfrau, Cologne, Germany), equivalent to one vial, into each calf. Injections were performed using a 27 -gauge cannula, in a grid-like manner with 1 injection $/ \mathrm{cm}^{2}$, and approximately $0.3 \mathrm{ml} /$ injection point. Injections were performed twice with an interval of 6 weeks between. Clinical inspection after the first injection cycle revealed mild reddening and swelling of the injection sites for approximately 3 days. No further side effects were reported. Two days after the second injection, however, the patient described a more painful injection site on the right calf compared to the first treatment. Moderate edema and reddening were visible. To exclude thrombosis, duplex sonography was performed without finding any pathology. Blood parameters were within normal ranges; D-dimer testing was negative. The patient was treated with ibuprofen $4001-1-1$ for 3 days. Pain and swelling were rapidly reduced after 2 days. However, 4 weeks later the patient presented with a palpable, mobile nodule in the dorsal region of the right calf which was painful on palpation during clinical examination. The overlying skin looked normal, the nodule was not clinically apparent. Ultrasound control revealed a round to oval structure $(1.3 \times 2.0 \mathrm{~cm})$ in the subcutaneous fat surrounded by echo-dense parts, corresponding to fibrosis. Puncturing of the nodule revealed a yellowish fluid, corresponding to liquid fat necrosis. Further treatments with PPC injections were rejected. We recommended the patient to observe the lesion and wait for possible spontaneous resolving. However, after 4 months the nodule had not disappeared and was therefore excised under local anesthesia. Histopathology revealed a thick fibrous capsule with amounts of necrotic fat tissue inside. In between the fibrous tissue some giant cells were present. The diagnosis of encapsulated fat necrosis was confirmed. Wound healing was without further complication. No recurrence occurred until today, 3 months after extirpation.

Despite its broad usage, the precise action mechanism of Lipostabil still remains unclear $[2,3]$. It seems that the injected substance leads to a localized necrosis of fat tissue with a subsequent inflammatory cascade and initiation of a repair process [3]. Chronological evaluation of human fat after lipolysis with PPC has revealed that after the induced destruction of fat cells a neutrophilic infiltrate developed in early stages, followed by lymphocytic infiltration, followed by macrophages/lipohages with possible pseudocysts, and fibrosis as late stage finding of a repair process [3].

\section{KARGER}

(C) 2008 S. Karger AG, Basel

Fax +4161306 1234

E-Mail karger@karger.ch

www.karger.com
Accessible online at: www.karger.com/drm 
Encapsulated fat necrosis, also named 'nodular cystic fat necrosis', 'mobile encapsulated lipoma' or 'posttraumatic degeneration and herniation', was first described in 1975 by SchmidtHermes and Loskant [4]. It is characterized by solitary or multiple, subcutaneous nodules, mostly located on the lower extremity and consisting of degenerated or necrotic fat tissue encapsulated by thin to thick fibrous tissue. The exact causes remain unclear; however, trauma and subsequent interruption of blood supply are speculated to play a major role [5]. Histopathology shows a fibrous capsule with degenerated or necrotic fat tissue in between, sometimes accompanied by inflammation and calcification.

The synopsis of clinical aspect, ultrasound, aspirated tissue and histopathology confirmed the diagnosis of encapsulated fat necrosis in our patient. It seems that the injected PPC-containing substance led to fat necrosis with the subsequent formation of surrounding fibrosis [5]. The edema, typically induced by injection lipolysis, may also play an additional role, probably by reducing the local blood supply due to pressure. In our patient, further treatments with PPC were rejected due to the complication. Both physician and patient did not observe sufficient fat reduction after the performed 2 injections.

To the best of our knowledge, our patient is the first case to present encapsulated fat necrosis after injection lipolysis for the reduction of localized fat accumulations. We assume that encapsulated fat necrosis has to be seen as a possible complication of the nonsurgical procedure described above. Considering the supposed mechanism of action of PPC and the frequency of the procedure, we assume that encapsulated fat necrosis is underreported.

\section{References}

1 Bechara FG, Sand M, Sand D, Rotterdam S, Stucker M, Altmeyer P, Hoffmann K: Lipolysis of lipomas in patients with familial multiple lipomatosis: an ultrasound-controlled trial. J Cutan Med Surg 2006;10: $155-159$.

2 Rotunda AM, Suzuki H, Moy RL, Kolodney MS: Detergent effects of sodium deoxycholate are a major feature of an injectable phosphatidylcholine formulation used for localized fat dissolution. Dermatol Surg 2004;30:1001-1008.

3 Bechara FG, Sand M, Hoffmann K, Sand D, Altmeyer P, Stucker M: Fat tissue after lipolysis of lipomas: a histopathological and immunohistochemical study. J Cutan Pathol 2007;34:552-557.

4 Schmidt-Hermes HJ, Loskant G: Verkalkte Fettgewebsnekrose der weiblichen Brust. Med Welt 1975;26:1179-1180.

5 Kiryu H, Rikihisa W, Furue M: Encapsulated fat necrosis - A clinicopathological study of 8 cases and a literature review. J Cutan Pathol 2000;27:19-23.

Falk Georges Bechara, MD

Department of Dermatology and Allergology

Ruhr University Bochum, St. Josef Hospital

Gudrunstrasse 56, DE-44791 Bochum (Germany)

Tel. +49 234 5090, Fax +492345093445

E-Mailf.bechara@derma.de
Dermatology 2008;216:181-183

DOI: 10.1159/000111520

\section{Erythema-Multiforme-Like Eruption Recurring in Ultraviolet-Exposed Skin}

\author{
Risa Tamagawa-Mineoka, Norito Katoh, Koji Masuda, \\ Saburo Kishimoto
}

Department of Dermatology, Kyoto Prefectural University of Medicine Graduate School of Medical Science, Kyoto, Japan

Key Words

Erythema multiforme $\cdot$ Fixed drug eruption $\cdot$ Recall

phenomenon - Ultraviolet B

We report a case of an erythema multiforme (EM)-like eruption in a 48-year-old Japanese woman who had suffered from recurrent exudative erythema of the face, trunk and extremities for a year. Eruption seemed to recur at the same sites with ultraviolet (UV) exposure. In phototesting, UVB irradiation induced macules and papules extending beyond the exposed area in the back, and eruptions resembling the target lesions appeared in the trunk and extremities. The patient was diagnosed with EM-like eruption induced by UVB irradiation. Recently, cases of drug eruptions occurring in a photo-distributed pattern have been reported. In these reports, the involvement of photo recall phenomenon was discussed. EM-like eruption is known to be triggered by UV exposure, however, the mechanism has not been revealed yet. Our case suggests that EM-like eruption recurring at UV-exposed areas may be involved in recall phenomenon.

The patient was a 48-year-old Japanese woman who had suffered from recurrent exudative erythema of the face, trunk and extremities for a year. She had taken no medication during this period. Her medical history including drug allergy was insignificant. She had worked as an aromatherapist for a year. Eruptions often appeared within several hours after UV exposure on a sunny day and disappeared within about a week. Eruptions seemed to recur at the sites of UV exposure. These findings suggested an abnormal response to UV irradiation; therefore, phototesting was performed by illuminating the back with UVA $\left(5,10\right.$ and $\left.15 \mathrm{~J} / \mathrm{m}^{2}\right)$ and UVB (10 doses: $20-200 \mathrm{~mJ} / \mathrm{m}^{2}$ ) using a Dermaray M-DMR100. UVB-induced macules and papules extending beyond the exposed area appeared $24 \mathrm{~h}$ after UVB irradiation at doses above $160 \mathrm{~mJ} / \mathrm{m}^{2}$ (fig. 1), and eruptions resembling target lesions appeared at the same site as previous eruptions on the trunk and extremities (fig. 2). UVA irradiation did not cause erythematous lesions. To assess whether there was a possible allergy to essential oils, patch and photopatch testing was performed. All test reactions were negative. Despite careful history-taking, other agents suspected to be causative were not found.

A skin biopsy specimen from the right forearm showed satellite cell necrosis of keratinocytes, lymphocytic infiltration around the upper dermal vessels and along the dermoepidermal junction, and edema of the papillary dermis. Laboratory findings were normal. Serological tests were negative for herpes simplex virus (HSV) and mycoplasma infection, and tests for antinuclear and anti-SS-A/B antibodies were also negative. The patient was diagnosed as having an erythema-multiforme (EM)-like eruption induced by UVB 